\title{
Undifferentiated sinonasal malignant melanoma: A case report
}

\author{
JUN DU* , LIANG-LIANG HUANG ${ }^{*}$, AO XU, AN-LI ZHANG, XUE KONG, MIN DING, WEN HU, ZHEN-LI GUO, \\ WEN ZHONG, SI-BAI SUN, HENG LI, JIE CHEN, QIAN SHEN, LU-LU XU and HAI-BO WU
}

Department of Pathology, Anhui Provincial Hospital, Hefei, Anhui 230001, P.R. China

Received September 29, 2016; Accepted January 12, 2018

DOI: $10.3892 / \mathrm{ol} .2018 .8662$

\begin{abstract}
Undifferentiated sinonasal malignant melanoma (MM) is a rare type of tumor, which can be easily misdiagnosed. The present study reports a 41-year-old male patient who presented with a 4-day history of epistaxis. Clinical examination and radiological imaging lead to the detection of a mass in the right sinonasal region. Histopathological examination revealed that the mass was composed of malignant epithelioid cells arranged in nests and sheets. These cells displayed a hemangiopericytoma-like pattern with antler-like branching vessels. Immunohistochemical staining revealed that the tumor cells exhibited negative expression of melanocytic markers. This increased the difficulty of distinguishing undifferentiated MM from other malignant tumors located in the sinonasal area, particularly undifferentiated nasopharyngeal carcinoma. The diagnosis of undifferentiated MM was determined by ultrastructures, including the mature melanosomes and premelanosomes, in tumor cells by transmission electron microscopy. The present study suggests that the analysis of cancer stem cell marker and vasculogenic mimicry may be an important auxiliary tool for the diagnosis of MM.
\end{abstract}

\section{Introduction}

Malignant melanoma (MM) is a type of tumor arising from melanocytes, which originate from neural crest cells (1). The common primary sites of MM are the skin and mucosal surfaces (2). MM is associated with a very high mortality rate and an extremely poor prognosis (3). Therefore, the accurate

Correspondence to: Dr Hai-Bo Wu, Department of Pathology, Anhui Provincial Hospital, 17 Lujiang Road, Hefei, Anhui 230001, P.R. China

E-mail: bbwuhaibo@sina.com

${ }^{*}$ Contributed equally

Abbreviations: MM, malignant melanoma; MRI, magnetic resonance imaging; VM, vasculogenic mimicry; H\&E, hematoxylin and eosin; SMA, smooth muscle actin; Fli-1, friend leukemia integration 1 transcription factor; EMA, epithelial membrane antigen

Key words: melanoma, undifferentiated, diagnosis, pathology diagnosis of $\mathrm{MM}$ is essential for providing appropriate and timely treatment. Sinonasal MM accounts for $<1 \%$ of all melanomas and $<5 \%$ of all sinonasal tract neoplasms (4). Patients with sinonasal MM often display non-specific symptoms, including nasal obstruction or epistaxis, causing clinical misdiagnosis (1). Based on melanin pigmentation, MM can be histopathologically categorized into melanotic and amelanotic subtypes $(1,5)$. It is difficult to diagnose amelanotic MM in undifferentiated tumor cells as they exhibit negative expression of all melanocytic markers (6). The present study reports a case of undifferentiated sinonasal MM, which mimics a poorly differentiated carcinoma with aberrant expression of epithelial markers. The ultrastructural characteristics of the tumor cells and the tumor phenotypes, including the proportion of cancer stem cells and vaculogenic mimicry (VM), were determined.

\section{Case report}

Written informed consent was acquired from the patient discussed in the present report, and the following study was ethically approved by the Ethics Committee of the Anhui Provincial Hospital (Anhui, China). A 41-year-old male patient was admitted to Anhui Provincial Hospital (Hefei, China) in July 2016. The patient reported a month of congestion of the right nasal passage with no rhinorrhea or bleeding until the 4 days prior to presentation, in which progressive epistaxis was experienced. Magnetic resonance imaging (MRI) revealed a lesion in the right nasal cavity and ethmoid sinus (Fig. 1A and B). Nasal endoscopic examination indicated a red mass occupying the right side of the nasal cavity and the middle meatus. The mass had invaded the middle turbinate, nasal septum and sieve plate, and bone destruction had occurred. Complete excision of the primary lesion with at least $1.5 \mathrm{~cm}$ of normal tissue was performed, and a sentinel lymph node biopsy achieved a negative result. The otolaryngologist identified the surface of the mass to be rough, brittle and to bleed easily when palpated. The patient exhibited stage II disease at diagnosis, and radiotherapy was indicated.

The specimen was examined by two pathologists. The excised tissue was fixed in $10 \%$ neutral-buffered formalin for $10 \mathrm{~h}$ at room temperature, then tissue samples were dehydrated in an ethanol/xylene series, embedded using fresh paraffin wax and maintained at $55-60^{\circ} \mathrm{C}$. Tissue samples were serially sectioned (4- $\mu$ m thick); 4 underwent hematoxylin and eosin (H\&E) staining and 23 underwent immunohistochemical staining. Immunohistochemistry 
was performed using the ChemMate Envision kit including secondary antibody (cat. no., DAKOK500711; Dako; Agilent Technologies, Inc., Santa Clara, CA, USA), according to the manufacturer's protocol (7). Sections were deparaffinized in xylene and dehydrated with descending alcohol series. Endogenous peroxidase was blocked with $0.1 \%$ hydrogen peroxide-methanol for $30 \mathrm{~min}$ at room temperature. Sections were washed once with PBS, and then microwaved for $15 \mathrm{~min}$ in $0.05 \mathrm{~mol}$ Tris buffer ( $\mathrm{pH}$ 9.0) for antigen retrieval, followed by washing three times with $\mathrm{PBS}$, and then incubated with primary antibodies at $4^{\circ} \mathrm{C}$ overnight. Following washing three times with PBS, they were incubated with horseradish peroxidase-conjugated dextran polymer reagent (dilution, 1:2,000; cat. no., sc-2004; Santa Cruz Biotechnology, Inc.) for $1 \mathrm{~h}$ at room temperature. The sections were incubated with the following 23 primary antibodies: Human melanoma black-45 (mouse monoclonal; cat. no., MAB-0098), Melan-A (mouse monoclonal; cat. no., MAB-0275), smooth muscle actin (SMA; mouse monoclonal; cat. no., Kit-0006), marker of proliferation Ki67 (mouse monoclonal; cat. no., Kit-0005), synaptophysin (Syn; rabbit monoclonal; cat. no., Kit-0022), cluster of differentiation 31 (CD31; mouse monoclonal; cat. no., MAB-0031), friend leukemia integration 1 transcription factor (Fli-1; mouse monoclonal; cat. no., MAB-0649), cluster of differentiation 21 (CD21; rabbit monoclonal; cat. no., RMA-0647), factor VIII (rabbit polyclonal; cat no., RAB-0070; all from Fuzhou Maixin Biotechnology Development Co., Ltd., Fuzhou, China). The following antibodies were purchased from ZSGB-BIO (Beijing, China): pan-cytokeratin (mouse monoclonal; cat. no., ZM-0069), vimentin (mouse monoclonal; cat. no., ZM-0260), cytokeratin 5 (rabbit monoclonal; cat. no., ZA-0518), CD34 (mouse monoclonal; cat. no., ZM-0046), epithelial membrane antigen (EMA; mouse monoclonal; cat. no., ZM-0095), S100 (rabbit polyclonal; cat. no., ZA-0225), cluster of differentiation 45 (CD45; mouse monoclonal; cat. no., ZM-0183), p40 (mouse monoclonal; cat. no., ZM-0472), sex determining region Y-box 10 (SOX10; rabbit monoclonal; cat. no., ZA-0624). Primary antibodies (volume, $100 \mu \mathrm{l}$ ) were provided as ready-to-use products and did not require further dilution. The cancer stem cells markers used include the following primary antibodies: Oct4 (rabbit polyclonal; dilution, 1:200; cat. no., 2750), sex determining region Y-box 2 (SOX2; rabbit monoclonal; dilution, 1:100; cat. no., 3579), Nanog (rabbit monoclonal; dilution, 1:800; cat. no., 4903; all from Cell Signaling Technology, Inc., Danvers, MA, USA), cluster of differentiation 133 (CD133; rabbit polyclonal; dilution, 1:100; cat. no., sc-30220; Santa Cruz Biotechnology, Inc., Dallas, TX, USA) and Nestin (mouse monoclonal; dilution, 1:200; cat. no., MAB-0566; Fuzhou Maixin Biotechnology).

Gross pathological examination resulted in the description of a grey irregular specimen of $5 \times 5 \times 1 \mathrm{~cm}^{3}$ and moderate hardness. Histopathologically, the mass was composed of malignant epithelioid cells arranged in nests and sheets (Fig. 2A). The overall cellularity was moderate to high with evident atypia. The tumor cells displayed a hemangiopericytoma-like pattern accompanied by antler-like branching vessels. The tumorstroma was focally desmoplastic and necrosis was not evident. Under a light microscope at x400, magnification (Leica 2500 microscope; Leica Microsystems, Inc., Buffalo Grove, IL, USA), the tumor cells demonstrated relatively uniform hyperchromatic, rounded nuclei with prominent nucleoli, and mitotic activity was conspicuous. The cytoplasm of the tumor was lightly eosinophilic to amphophilic, containing numerous vacuoles but exhibiting no pigment deposition (Fig. 2B).

Immunohistochemical analyses demonstrated that the tumor cells did not express melanocytic markers HMB-45, Melan-A, S100 or SOX10 (Fig. 3A). However, the tumor cells did express vimentin (Fig. 3B), EMA (Fig. 3C) and Fli-1 (Fig. 3D). Pan-cytokeratin expression demonstrated scattered expression among tumor cells (Fig. 3E). The tumor cells did not express cytokeratin 5, CD31, CD34, Syn, CD45, CD21, SMA, Factor VIII, p63 or p40. A total of $80 \%$ of the tumor cells were positive for Ki-67 staining (Fig. 3F). Ultrastructural analysis by transmission electron microscopy was performed to determine the malignancy type. The results demonstrated that mature melanosomes and premelanosomes existed in tumor cells (Fig. 4), supporting the diagnosis of MM.

In order to further understand the nature of undifferentiated MM, cancer stem cell marker expression was also analyzed, including that of CD133, SOX2, OCT4 and Nanog. The tumor cells expressed CD133 strongly and diffusely (Fig. 5A). SOX2 was expressed focally around the vessels (Fig. 5B), while nestin expression was negative for tumor cells, but positive for vascular mural cells (Fig. 5C). The tumor cells exhibited negative expression of OCT4 and Nanog. In order to investigate VM, CD34 and periodic acid-Schiff (PAS; B24200-250; Thermo Fisher Scientific, Inc., Waltham, MA, USA) double-staining was used to distinguish microvessels from VM. Sections were exposed to sodium periodate for $10 \mathrm{~min}$ following immunohistochemical staining of CD34 at room temperature, then rinsed with distilled water for $5 \mathrm{~min}$, followed by incubation with $0.1 \%$ Periodic acid-Schiff for $15 \mathrm{~min}$ at room temperature. All sections were counterstained with Mayer's hematoxylin for $2 \mathrm{~min}$ at room temperature, dehydrated with descending alcohol series, and mounted. VM was characterized as a channel positive for PAS without exhibiting positive CD34 staining of the endothelium (Fig. 5D).

\section{Discussion}

Primary MM of the sinonasal mucosa is a rare disease, with an equal incidence in males and females. It often occurs at 60-70 years of age, but rarely prior to 40 years of age (8). Obstruction or epistaxis are the most common early symptoms of sinonasal MM (1). Sinonasal MM has a high rate of local recurrence and distant metastasis $(1,3)$. According to previous literature, the 5-year survival rate of patients is $44-88.7 \%$ (9). One previous study revealed that the majority of patients with sinonasal MM exhibiting stage I or II disease demonstrated no evidence of recurrence after a follow-up period of 13.9 years. However, all patients presenting with a stage III diagnosis had succumbed to the disease during the follow-up period (10). The survival rate was associated with tumor location, tumor spread, tumor thickness, lymph node and distant metastases $(9,10)$. Therefore, the early and accurate diagnosis of sinonasal MM is essential for effective treatment of the disease.

Histopathologically, melanoma is categorized into melanotic and amelanotic types according to melanin 

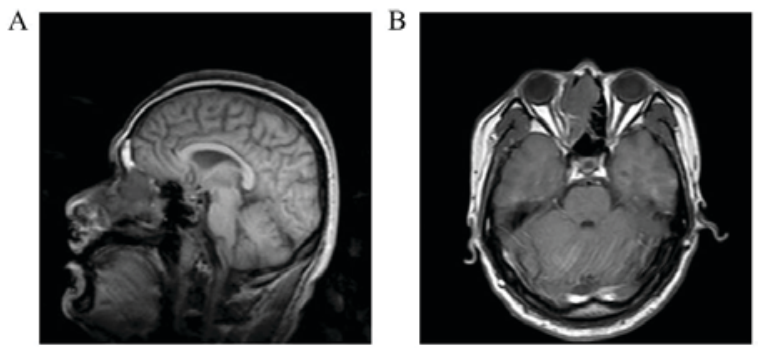

Figure 1. Preoperative magnetic resonance imaging. (A) Sagittal view and (B) axial view, demonstrating a mass occupying the right side of the nasal cavity, extending forward toward the right ethmoid sinus with erosion of adjacent bone tissues.
A

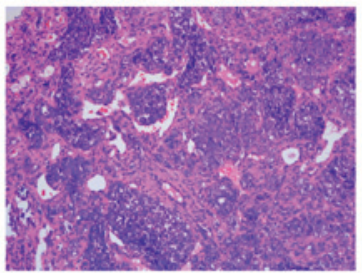

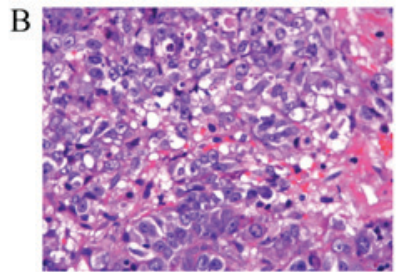

Figure 2. Histopathological analysis of the tumor tissue. (A) H\&E staining revealed that the tumor exhibited a nests and sheets arrangement, and displayed a hemangiopericytoma-like pattern with antler-like branching vessels (magnification, x100). (B) Higher magnification (x400) revealed that the tumor was composed of uniform epithelioid cells, with hyperchromatic, rounded or ovoid nuclei with prominent nucleoli and high mitotic activity. $\mathrm{H} \& \mathrm{E}$, hematoxylin and eosin.
A

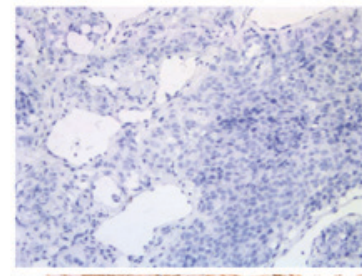

C

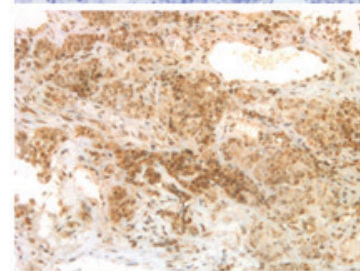

E

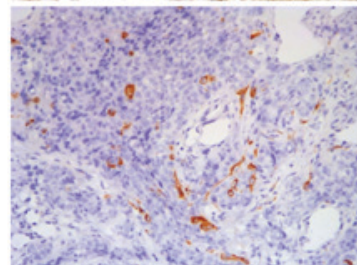

$\mathrm{B}$

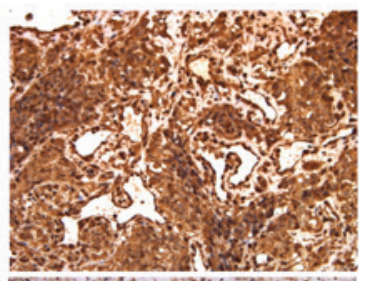

D

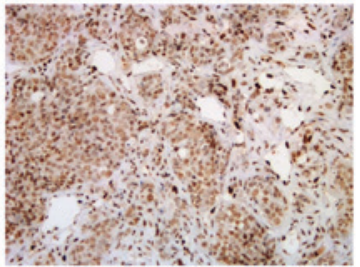

$\mathrm{F}$

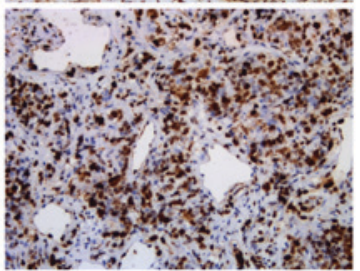

Figure 3. Immunohistochemical analysis of undifferentiated malignant melanoma. (A) The tumor cells did not express human melanoma black-45. (B) The tumor cells were positive for vimentin expression. (C) The tumor cells were positive for epithelial membrane antigen expression. (D) The tumor cells were positive for friend leukemia integration 1 transcription factor nucleic expression. (E) The tumor cells exhibited scattered expression of pan-cytokeratin. (F) Ki-67 expression was positive in the nuclei of $80 \%$ tumor cells. Magnification, x200.

pigmentation (11). MM tumors are composed of a variety of cell types: Epithelioid, spindled, clear-cell, plasmacytoid and

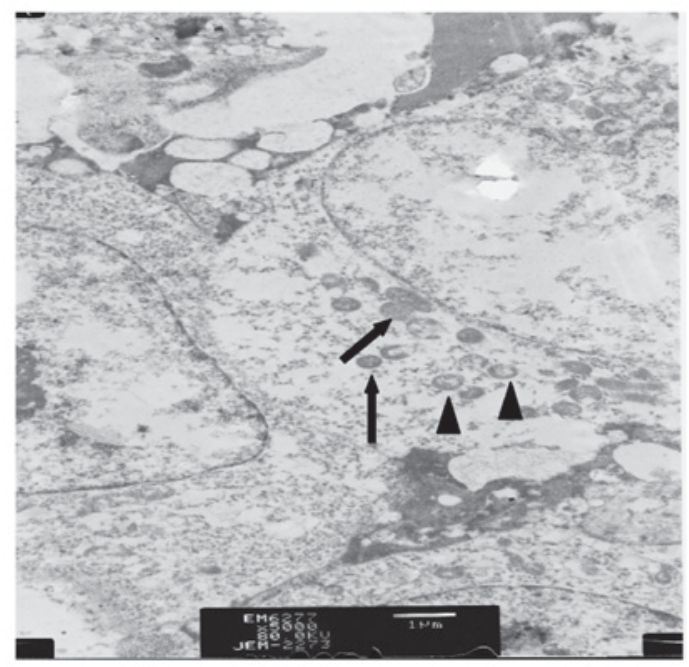

Figure 4. The ultrastructure of the undifferentiated malignant melanoma. Transmission electron microscopy detected a small number of mature melanosomes, indicated by black arrows, and premelanosomes, indicated by black arrowheads, within the tumor cells (scale bar: $1 \mu \mathrm{m}$ ).
A

$\mathrm{C}$
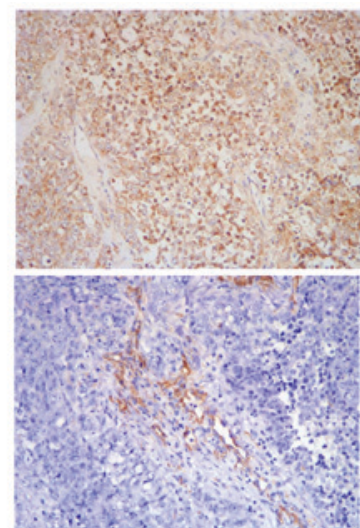

B

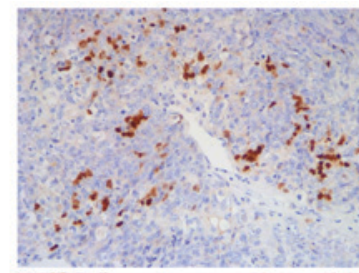

D

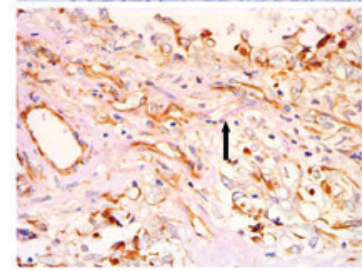

Figure 5. Cancer stem cells markers and vasculogenic mimicry detection in undifferentiated malignant melanoma. (A) The tumor cells were positive for CD133 expression (magnification, x200). (B) The tumor cells exhibited scattered expression of sex determining region Y-box 2 (magnification, x200). (C) The tumor cells did not express Nestin, but did exhibit vascular mural cells (magnification, x200). (D) CD34/PAS double staining revealed vasculogenic mimicry positive for PAS staining (purple-red) and negative for CD34 (black arrow). The microvessels were positive for both CD34 and PAS staining (magnification, $x 400$ ). CD133, cluster of differentiation 133; CD34, cluster of differentiation 34; PAS, periodic acid-Schiff.

mixed-cell (12). In a number of cases, the melanoma cells may be undifferentiated with no expression of melanocytic markers, resulting in difficulty regarding its diagnosis $(6,13)$.

In the present case report, the tumor cells exhibited negative expression of melanocytic markers, including HMB-45, Melan-A, S100 and SOX10. However, the tumor cells exhibited positive expression of epithelial markers, including EMA and pan-cytokeratin. These characteristics mimic those of undifferentiated nasopharyngeal carcinomas that occur commonly in sinonasal regions (14). However, characteristically, undifferentiated nasopharyngeal carcinomas exhibit cytokeratin 5, p63 and p40 expression (15), which was not exhibited by the tissue analyzed in the present study. On the other hand, expression of the endothelial cell 
marker, Fli-1, was observed, which raised the possibility of epithelioid angiosarcoma, characterized by Fli-1, CD31, CD34 and factor VIII expression (16). However, a previous study demonstrated that Fli-1 may be strongly expressed in MM, and that Fli-1 expression is associated with tumor cell proliferation rate and other aggressive behaviors (17). The immunohistochemical panel of the present study excluded other possible tumor types of the sinonasal region, including sinonasal neuroendocrine carcinoma, paraganglioma, olfactory neuroblastoma, pituitary adenoma, tumors of the Ewing family and follicular dendritic cell sarcoma. Sinonasal neuroendocrine carcinoma, paraganglioma, olfactory neuroblastoma, pituitary adenoma and tumors of the Ewing family which usually express Syn; however this was not expressed in the present case study. Follicular dendritic cell sarcoma demonstrated diffuse staining with CD21, while MM was negative for CD21. The results of transmission electron microscopy analysis revealed the presence of mature melanosomes and premelanosomes in the cytoplasm of the tumor cells, which are key ultrastructures of MM cells (18).

SOX2, CD133 and Nestin have been reported as cancer stem cell markers in melanoma (19-21). In the present study, the tumor cells of undifferentiated MM expressed CD133 and SOX2. Nestin was not expressed in tumor cells but was expressed in vascular mural cells. It is speculated that cancer stem cells may transdifferentiate into pericytes and participate in blood vessel remodeling $(22,23)$. Tumor cell vasculogenic mimicry (VM) refers to the plasticity of cancer cells forming de novo vascular networks, and is associated with malignant phenotypes and a poor prognosis $(24,25)$. Cancer stem cells may contribute to the formation of VM (26). VM was originally identified in malignant melanoma, and was subsequently demonstrated to be an important morphological feature of MM (27). In the present study, VM is detectable in undifferentiated MM due to stemness, which may be a useful tool for MM diagnosis.

The present study presents a rare case of undifferentiated sinonasal MM. Given that an accurate diagnosis of this type of tumor is challenging, it is demonstrated that the combination of a panel of conventional immunohistochemical markers, ultrastructure identification by transmission electron microscopy and the VM detection may be used to validate the diagnosis of $\mathrm{MM}$, and to exclude other malignancies of the sinonasal area.

\section{Acknowledgements}

Not applicable.

\section{Funding}

No funding was received.

\section{Availability of data and materials}

The datasets used and/or analyzed during the current study are available from the corresponding author on reasonable request.

\section{Authors' contributions}

JD and LLH contributed to the design of the research, drafting the manuscript, gave final approval of the version to be published, and agreed to be accountable for all aspects of the work in ensuring that questions related to the accuracy or integrity of any part of the work are appropriately investigated and resolved. AX, ALZ, XK, MD, WH and ZLG performed the experiments. WZ, SBS and HL analyzed the data. JC and QS made substantial contributions to conception and acquisition of data. LLX made contributions to interpretation of data. HBW contributed to the design of the research, and revised and finalized the article. All authors read and approved the final manuscript.

\section{Ethics approval and consent to participate}

This study was ethically approved by the Ethics Committee of the Anhui Provincial Hospital (Anhui, China). Informed consent was obtained from the study participant.

\section{Consent for publication}

All patients provided consent for the publication of their data

\section{Competing interests}

The authors declare that they have no competing interests.

\section{References}

1. Zhu W, Zou B and Wang S: Clinicopathological features and prognosis of sinonasal mucosal malignant melanoma: A retrospective study of 83 cases in a Chinese population. ORL J Otorhinolaryngol Relat Spec 78: 94-104, 2016.

2. Safadi RA, Bader DH, Abdullah NI and Sughayer MA: Immunohistochemical expression of keratins $6,7,8,14,16,18$, 19 , and MNF-116 pancytokeratin in primary and metastatic melanoma of the head and neck. Oral Surg Oral Med Oral Pathol Oral Radiol 121: 510-519, 2016.

3. Bradley PJ: Primary malignant mucosal melanoma of the head and neck. Curr Opin Otolaryngol Head Neck Surg 14: 100-104, 2006.

4. Smith SM, Schmitt AC, Carrau RL and Iwenofu OH: Primary sinonasal mucosal melanoma with aberrant diffuse and strong desmin reactivity: A potential diagnostic pitfall! Head Neck Pathol 9: 165-171, 2015.

5. Thomas NE, Kricker A, Waxweiler WT, Dillon PM, Busman KJ, From L, Groben PA, Armstrong BK, Anton-Culver H, Gruber SB, et al: Comparison of clinicopathologic features and survival of histopathologically amelanotic and pigmented melanomas: A population-based study. JAMA Dermatol 150: 1306-1314, 2014

6. Agaimy A, Specht K, Stoehr R, Lorey T, Märkl B, Niedobitek G, Straub M, Hager T, Reis AC, Schilling B, et al: Metastatic malignant melanoma with complete loss of differentiation markers (undifferentiated/dedifferentiated melanoma): Analysis of 14 patients emphasizing phenotypic plasticity and the value of molecular testing as surrogate diagnostic marker. Am J Surg Pathol 40: 181-191, 2016.

7. Liu J, Wang Y, Liu Y, Liu Z, Cui Q, Ji N, Sun S, Wang B, Wang Y, Sun $X$ and Liu Y: Immunohistochemical profile and prognostic significance in primary central nervous system lymphoma: Analysis of 89 cases. Oncol Lett 14: 5505-5512, 2017.

8. Montone KT: The diferential diagnosis of sinonasal/nasopharynheal neuroendocrine/neuroectodermally derived tumors. Arch Pathol Lab Med 139: 1498-1507, 2015.

9. Houette A, Gilain L, Mulliez A, Mom T and Saroul N: Prognostic value of two tumour staging classifications in patients with sinonasal mucosal melanoma. Eur Ann Otorhinolaryngol Head Neck Dis 133: 313-317, 2016.

10. Thompson LD, Wieneke JA and Miettinen M: Sinonasal tract and nasopharyngeal melanomas: A clinicopathologic study of 115 cases with a proposed staging system. Am J Surg Pathol 27: 594-611, 2003. 
11. Teixeira TF, Gentile LB, da Silva TC, Mennecier G, Chaible LM, Cogliati B, Roman MA, Gioso MA and Dagli ML: Cell proliferation and expression of connexins differ in melanotic and amelanotic canine oral melanomas. Vet Res Commun 38: 29-38, 2014.

12. Kerr EH, Hameed O, Lewis JS Jr, Bartolucci AA, Wang D and Said-AI-Naief N: Head and neck mucosal malignant melanoma: Clinicopathologic correlation with contemporary review of prognostic indicators. Int J Surg Pathol 20: 37-46, 2012.

13. Jalas JR, Vemula S, Bezrookove V, Leboit PE, Simko JP and Bastian BC: Metastatic melanoma with striking adenocarcinomatous differentiation illustrating phenotypic plasticity in melanoma. Am J Surg Pathol 35: 1413-1418, 2011.

14. Franchi A: An update on sinonasal round cell undifferentiated tumors. Head Neck Pathol 10: 75-84, 2016.

15. Singh L, Ranjan R, Arava S and Singh MK: Role of p40 and cytokeratin 5/6 in the differential diagnosis of sinonasal undifferentiated carcinoma. Ann Diag Pathol 18: 261-265, 2014.

16. Folpe AL, Chand EM, Goldblum JR and Weiss SW: Expression of Fli-1, a nuclear transcription factor, distinguishes vascular neoplasms from potential mimics. Am J Surg Pathol 25: 1061-1066, 2001.

17. Torlakovic EE, Slipicevic A, Flørenes VA, Chibbar R, DeCoteau JF and Bilalovic N: Fli-1 expression in malignant melanoma. Histol Histopathol 23: 1309-1314, 2008.

18. Ordóñez NG and Mackay B: Electron microscopy in tumor diagnosis: Indications for its use in the immunohistochemical era. Hum Pathol 29: 1403-1411, 1998.

19. Wang J, Ding N, Li Y, Cheng H, Wang D, Yang Q, Deng Y, Yang Y, Li Y, Ruan X, et al: Insulin-like growth factor binding protein 5 (IGFBP5) functions as a tumor suppressor in human melanoma cells. Oncotarget 6: 20636-20649, 2015.
20. Zimmerer RM, Korn P, Demougin P, Kampmann A, Kokemüller H, Eckardt AM, Gellrich NC and Tavassol F: Functional features of cancer stem cells in melanoma cell lines. Cancer Cell Int 13: 78, 2013.

21. Kuk SK, Won CH, Lee WJ, Shin WJ, Yoon HJ, Hong SD, Hong SP and Lee J II: Prognostic significance of nestin in primary malignant melanoma of the oral cavity. Melanoma Res 26: 457-463, 2016.

22. Klein D, Meissner N, Kleff V, Jastrow H, Yamaguchi M, Ergün S and Jendrossek V: Nestin (+) tissue-resident multipotent stem cells contribute to tumor progression by differentiating into pericytes and smooth muscle cells resulting in blood vessel remodeling. Front Oncol 4: 169, 2014.

23. Cheng L, Huang Z, Zhou W, Wu Q, Donnola S, Liu JK, Fang X, Sloan AE, Mao Y, Lathia JD, et al: Glioblastoma stem cells generate vascular pericytes to support vessel function and tumor growth. Cell 153: 139-152, 2013.

24. Maniotis AJ, Folberg R, Hess A, Seftor EA, Gardner LM, Pe'er J, Trent JM, Meltzer PS and Hendrix MJ: Vascular channel formation by human melanoma cells in vivo and in vitro: Vasculogenic mimicry. Am J Pathol 155: 739-752, 1999.

25. Spiliopoulos K, Peschos D, Batistatou A, Ntountas I, Agnantis N and Kitsos G: Vasculogenic mimicry: Lesson from melanocytic tumors. In Vivo 29: 309-317, 2015.

26. Wang SS, Gao XL, Liu X, Gao SY, Fan YL, Jiang YP, Ma XR, Jiang J, Feng H, Chen QM, et al: CD133+ cancer stem-like cells promote migration and invasion of salivary adenoid cystic carcinoma by inducing vasculogenic mimicry formation. Oncotarget 7: 29051-29062, 2016.

27. Hendrix MJ, Seftor EA, Seftor RE, Chao JT, Chien DS and Chu YW: Tumor cell vascular mimicry: Novel targeting opportunity in melanoma. Pharmacol Ther 159: 83-92, 2016. 